\author{
Marco Trucco \\ Piero Meineri \\ Luigi Ruiz \\ on behalf of the "Gruppo \\ Neurologico Ospedaliero \\ Interregionale per lo Studio \\ delle Cefalee" (Neurological \\ Hospital Interregional \\ Group for the Study of \\ Headaches)
}

\section{Preliminary results of a withdrawal and detoxification therapeutic regimen in patients with probable chronic migraine and probable medication overuse headache}

Published online: 20 July 2005

\begin{abstract}
Chronic migraine (CM) is an invalidating condition affecting a significant population of headache sufferers, frequently associated with medication overuse headache $(\mathrm{MOH})$. Controlled trials and guidelines for the treatment of $\mathrm{MOH}$ are currently not available. We studied the efficacy of a therapeutic regimen for the withdrawal of the overused drug and detoxification in a sample of patients suffering from probable $\mathrm{CM}$ and probable $\mathrm{MOH}$ during admission in eight hospitals of Piemonte-Liguria-Valle d'Aosta. Fifty patients, 42 females (84\%) and 8 males $(16 \%)$, mean age at observation $50.66 \pm 13.08$ years, affected by probable $\mathrm{CM}$ and daily medication overuse following IHS diagnostic criteria were treated as inpatients or in a day hospital. Headache index (HI) and daily drug intake (DDI) were used for evaluating the severity of headache and medication overuse. The patients were treated by abrupt discontinuation of the overused drug and by a therapeutic protocol including i.v. hydration, dexamethasone, metoclopramide and benzodiazepines for 7-10 days. Prophylactic medication was started immediately after admission. Analgesics or triptans were used under medical control only in cases of severe rebound headache. Diagnostic protocol
\end{abstract}

included routine blood tests (at admission and at discharge), dosage of B12 and folic acid. Patients underwent follow-up controls one, three and six months after discharge. The initial diagnosis was probable $\mathrm{CM}$ in almost all patients included in the study (41 patients); in nine patients the diagnosis was not specified (coded only as $\mathrm{CDH}$ ). The overused medications were simple analgesics in 17 cases (34\%), combination analgesics in 19 cases (38\%), triptans alone or with analgesics in 13 cases $(26 \%)$ and ergotamine in 2 cases $(4 \%)$. We collected data from 39 patients at first follow-up (1 month), 32 after 3 months and 14 after 6 months. Mean HI was 0.91 at admission, 0.22 at discharge, 0.38 after 30 days, 0.46 after 3 months and 0.48 after 6 months. Mean DDI was 2.80 at admission, 0.39 at discharge, 0.41 after 1 month, 0.52 after 3 months and 0.59 after 6 months. These results are on average positive and tend to remain stable with time. Although preliminary and obtained on a limited number of patients at 6month follow-up, our results seem to be encouraging about the use of the proposed therapeutic protocol.

Key words Chronic daily headache • Chronic migraine $\cdot$ Medication overuse - Detoxification 


\section{Introduction}

Chronic daily headache $(\mathrm{CDH})$ is one of the main therapeutic problems within primary headaches. Chronic migraine (CM) (following ICHD-II: 1.5.1 [1]), is one of the most frequent causes of $\mathrm{CDH}$.

Chronic abuse of ergotamine [2], analgesics [3], opioids [4] and triptans [2-5] may lead to medicationoveruse headache $(\mathrm{MOH})$, a secondary headache frequently associated with $\mathrm{CDH}$. Abrupt withdrawal of ergotamine, analgesics and triptans [6] can also cause rebound headache. The treatment of $\mathrm{CDH}$ and $\mathrm{MOH}$ includes the withdrawal of the abused drug [7]. Further, some therapeutic protocols were proposed [8, 9] but controlled trials and guidelines are currently not available. Hospitalisation is recommended by several authors [10], but without general consent [11].

We studied the efficacy of a withdrawal and detoxification therapeutic regimen in 50 patients suffering from probable $\mathrm{CM}$ and probable $\mathrm{MOH}$, performed within eight hospital headache centres. This protocol was followed by a six-month follow-up.

\section{Materials and methods}

Fifty patients, 42 females (84\%) and eight males (16\%), mean age at observation $50.66 \pm 13.08$ years (range $24-76$ ), affected by probable CM (ICHD-II 1.6.5) and probable MOH (ICHD-II 8.2.7) underwent a withdrawal and detoxification therapeutic regimen as inpatients $(25,50 \%)$ or in a day hospital $(25,50 \%)$.

Inclusion criteria were:

1. age $\geq 18$ years;

2. diagnosis of $\mathrm{CDH}$;

3. headache index $(\mathrm{HI}) \geq 0.7$;

4. daily drug intake (DDI) $\geq 1$;

5. the above conditions having lasted for at least 6 months;

6. verbal informed consent.

The patients were enrolled after a clinical neurological evaluation by an experienced headache specialist including diagnosis and time patterns of primary headache, comorbidity, abused drug, previous prophylaxes and neurological examination. HI and DDI were used for evaluating the severity of headache and medication overuse within a one-month run-in period (T0). These data were collected mostly as anamnestic data; some of the patients filled out a headache diary. Clinical characteristics of the population are shown in Table 1.

At admission (as inpatients or in a day hospital, according to severity of the headache, amount and type of abused drug, and preference of the patient), they underwent a diagnostic protocol including blood tests (at admission and at discharge), dosage of B12 and folic acid.

The treatment included:

1. Immediate withdrawal of all abused drugs;

2. i.v. hydration (1000-1500 cc of saline solution);

3. i.v. anxiolytic therapy (i.e., clordemetildiazepam 1-3 mg daily or diazepam $5-15 \mathrm{mg}$ daily);

4. i.v. dexamethasone ( $8 \mathrm{mg}$ daily);

5. i.v. metoclopramide (10 $\mathrm{mg}$ daily).

In case of severe headache attacks, only single drugs were allowed (triptans, $1 \mathrm{cp}$. daily; or lysine acetylsalicylate, $1000 \mathrm{mg}$ daily), and always different from the abused drug. Eight patients (16\%) took butalbital-containing preparations (mean daily dose $306.25 \mathrm{mg}$ ), but none of them presented symptoms of dependence with need of phenobarbital therapy. Duration of treatment could vary from nine to fifteen days. Prophylaxis was continued if present at the beginning of treatment; if not, it was immediately started. All patients were instructed about the positive effects of behavioural modifications. Follow-up data were collected by means of a headache diary.

Patients were evaluated before (T0) and after (T10) our therapeutic regimen. They underwent follow-up controls one (T30), three (T90) and six months (T120) after discharge. Due to the limited number of patients seen at the six-month follow-up visit, we restricted the evaluation of results to the 32 patients seen at the three-month follow-up visit. Statistical evaluation was performed on HI and DDI using Student's $t$-test $v s$. T0 data.

\section{Results}

The initial diagnosis was probable chronic migraine (1.6.5) in $41 / 50$ patients; in 9 patients the diagnosis was only coded as $\mathrm{CDH}$. The overused medications were simple analgesics/NSAIDs in 14 cases (28\%), analgesics/NSAIDs in combination in 22 cases $(44 \%)$, triptans alone in 6 cases

Table 1 Patients' characteristics at observation. Data are expressed as mean \pm SD (range) except otherwise indicated

\begin{tabular}{llll}
\hline & In-patients & Day hospital & Total \\
\hline Males, $n$ & 3 & 5 & 8 \\
Females, $n$ & 22 & 20 & 42 \\
Mean age at observation, years & $49.12 \pm 12.85(24-74)$ & $52.20 \pm 13.39(29-76)$ & $50.66 \pm 13.08(24-76)$ \\
Length of overuse, years & $4.41 \pm 8.10(6 \mathrm{~m}-30 \mathrm{y})$ & $1.80 \pm 3.53(6 \mathrm{~m}-15 \mathrm{y})$ & $3.41 \pm 6.27(6 \mathrm{~m}-30 \mathrm{y})$ \\
Headache index & $0.94 \pm 0.11(0.7-1)$ & $0.89 \pm 0.14(0.7-1)$ & $0.91 \pm 0.13(0.7-1)$ \\
Daily drug intake & $3.12 \pm 2.07(1-10)$ & $2.48 \pm 1.39(1-6)$ & $2.80 \pm 1.77(1-10)$ \\
\hline
\end{tabular}


$(12 \%)$ or in association with analgesics/NSAIDs in 6 cases (12\%) and ergot derivatives (in combination) in 2 cases $(4 \%)$. Nineteen $(38 \%)$ of our patients took more than one preparation. Twenty-three patients (46\%) took caffeinecontaining preparations (mean daily dose $270 \mathrm{mg}$ ). None of our patients took opioid-containing preparations.

Comorbidity included anxious depression in 24 patients $(48 \%)$, arterial hypertension in 8 patients $(16 \%)$ and obesity in 3 patients $(6 \%)$. Only in one case gastric disorders were reported.

Previous prophylactic treatments were: tricyclic antidepressants in 18 cases; calcium antagonists in 15 cases; beta-blockers in 12 cases; SSRI in 8 cases; antiepileptic agents in 8 cases; pizotifen in 4 cases; other pharmacological prophylaxes in 5 cases; and non-pharmacological treatments in 2 cases (in 20 cases multiple prophylactic treatments were reported). Seventeen patients did not take any prophylaxis.

We found altered values of blood tests at the first visit only in a minority of patients: high cholesterol levels in 7 cases, alteration of hepatic enzymes in 2 cases, low folate values in 2 cases and mild hyperglycaemia in 1 case.

Three patients were early drop-outs and six were lost at follow-up. Preliminary data were collected from 39 patients after 1 month, 32 after 3 months and 14 after 6 months.

The suggested prophylaxis was: sodium valproate in 27 cases, amitriptyline in 6 cases, flunarizine in 6 cases, propranolol in 6 cases, SSRI in 5 cases, topiramate in 5 cases and other prophylaxes in 2 cases. In some cases we suggested a combined prophylaxis.

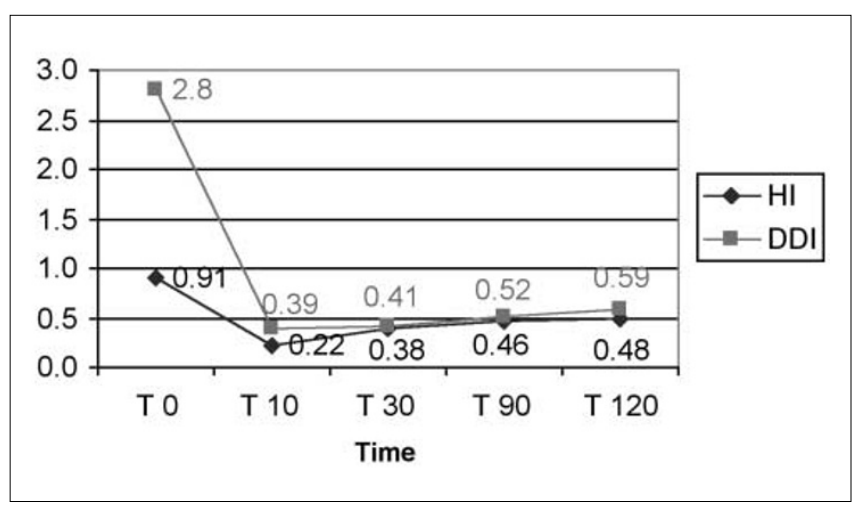

Fig. 1 Time course of HI and DDI
The time course of HI and DDI are shown in Figure 1. Statistical evaluation of preliminary data is shown in Table 2. However, data from these 32 patients do not substantially differ from those regarding all patients.

At the T30 visit, following the course of headache and medication overuse, we were able to modify the original diagnosis only in 26/32 patients, who withdrew drug overuse. In 23 of them headache significantly improved, allowing a diagnosis of MOH. The diagnosis was refined following the revision of criteria for 8.2 Medication overuse headache [12], so 4 were diagnosed as triptanoveruse headache (8.2.2), 3 as Analgesic-overuse headache (8.2.3), 9 as Combination analgesic-overuse headache (8.2.5), and 7 as Medication-overuse headache attributed to combination of acute medications (8.2.6). Three patients reported persistence of frequent migrainous attacks despite prophylaxis and so received a definite diagnosis of CM (ICHD-II 1.5.1). Six out of 32 patients continued medication overuse; their diagnosis remained probable $\mathrm{CM}$ and probable $\mathrm{MOH}$.

At T90, $19 / 32$ patients $(59.4 \%)$ experienced a significant relief from headache (below one half of the initial frequency), 24/32 (75.0\%) significantly reduced drug intake, and $8(25.0 \%)$ were therapeutic failures.

\section{Discussion}

Although only preliminary, our data are in accordance with previous observations [13] and are encouraging for the use of the protocol. The differences in HI and DDI before and after treatment are highly significant. These results tend to remain stable with time. We obtained also a good rate of positive individual results $(59.4 \%$ for headache relief and $75.0 \%$ for lowering drug intake).

It is difficult, due to the limited number of patients, to draw conclusions from these data. We found only a correlation between duration of overuse and unsuccessful outcome (patients with a successful outcome had overused medications for $1.71 \pm 2.27$ years; those with unsuccessful outcome had overused for $7 \pm 10.65$ years: $p<0.05)$. No correlation between type and amount of overused drug and successful outcome was found.

Table 2 Statistical evaluation (Student's $t$-test) on 32 patients at T0, T10, T30, T90

\begin{tabular}{|c|c|c|c|c|}
\hline & HI & $p v s . \mathrm{T} 0$ & DDI & $p v s . \mathrm{T} 0$ \\
\hline T0 & $0.94 \pm 0.11$ & & $3.03 \pm 1.96$ & \\
\hline T10 & $0.21 \pm 0.27$ & $<0.0001$ & $0.36 \pm 0.65$ & $<0.0001$ \\
\hline T30 & $0.38 \pm 0.34$ & $<0.0001$ & $0.40 \pm 0.58$ & $<0.0001$ \\
\hline T90 & $0.46 \pm 0.36$ & $<0.0001$ & $0.52 \pm 0.60$ & $<0.0001$ \\
\hline
\end{tabular}


The critical period for a good outcome in these patients is the first six months after detoxification.

We will continue the observation in the long-term following the promising results observed.

Acknowledgements We thank the following colleagues, members of the Neurological Hospital Interregional Group for the
Study of Headache, who participated in the study with the application of the protocol and follow-up observations: E. Grasso (Cuneo); M. Maggio (Ivrea); D. Ferrandi (Alessandria); E. Bottacchi, C. Lia (Aosta); E. Barbero (Biella); M. Fonzari, D. Farinini, D. Santoloci (Genova, S. Martino Hospital); F. Valguarnera (Genova, Sestri Ponente Hospital); M. Gionco, A. Febbraro (Torino); G. Perego (Pietra Ligure).

\section{References}

1. Headache Classification Subcommittee of the International Headache Society (2004) The International Classification of Headache Disorders, 2nd edn. Cephalalgia 24[Suppl 1]:1-160

2. Evers S, Gralow I, Bauer B et al (1999) Sumatriptan and ergotamine overuse and drug induced headache: a clinicoepidemiologic study. Clin Neuropharmacol 22:201-206

3. Granella F, Farina S, Malferrari G, Manzoni GC (1987) Drug abuse in chronic headache: a clinico-epidemiologic study. Cephalalgia 7:15-19

4. De Marinis M, Janiri L, Agnoli A (1991) Headache in the use and withdrawal of opiates and other associated substances of abuse. Headache 31:159-163

5. Limmroth V, Katsarava Z, Fritsche G, Diener HC (1999) Headache after frequent use of new 5-HT agonists zolmitriptan and naratriptan. Lancet 353:378-382
6. Katsarava Z, Fritsche G, Muessig M et al (2001) Clinical features of withdrawal headache following overuse of triptans and other headache drugs. Neurology 57:1694-1698

7. Hering R, Steiner TJ (1991) Abrupt outpatients withdrawal of medication in analgesic-abusing migraineurs. Lancet 337:1442-1443

8. Pini LA, Bigarelli M, Vitale G, Sternieri E (1996) Headaches associated with chronic use of analgesics: a therapeutic approach. Headache 36:433-439

9. Bonuccelli U, Nuti A, Lucetti C et al (1996) Amitriptyline and dexamethasone combined treatment in druginduced headache. Cephalalgia 16:197-200

10. Zed PJ, Loewen PS, Robinson G (1999) Medication-induced headache: overview and systematic review of therapeutic approaches. Ann Pharmacother 33:61-72
11. Suhr B, Evers S, Bauer B et al (1999) Drug-induced headache: long-term results of stationary versus ambulatory withdrawal therapy. Cephalalgia 19:44-49

12. Silberstein SD, Olesen J, Bousser M-G et al (2005) The International Classification of Headache Disorders, 2nd edn. (ICHD-II) - revision of criteria for 8.2 Medication-overuse headache. Cephalalgia 25:460-465

13. Baumgartner $C$, Wessely $P$, Bingöl $C$ et al (1989) Longterm prognosis of analgesic withdrawal in patients with druginduced headache. Headache 29:510-514 